\title{
EHER: An Energy Harvesting Aware Entropy-Based Routing Protocol in Mobile Ad-Hoc Wireless Sensor Networks
}

\author{
Kyuhyun Shim ${ }^{1}$, Kyusung Shim ${ }^{2}$, and Beongku An ${ }^{\circledR}$ \\ ${ }^{1,2}$ Dept. of Information System in Graduate School of Smart City Science \\ Management Hongik University, Republic of Korea \\ ${ }^{\circ}$ Dept. of Computer and Information Communications Engineering, \\ Hongik University, Republic of Korea \\ ${ }^{1}$ lread714@naver.com, ${ }^{2}$ kyusung@hongik.ac.kr, ${ }^{\circ}$ beongku@hongik.ac.kr
}

\begin{abstract}
In this paper, we propose an Energy Harvesting aware Entropy-based Routing protocol, called EHER, in mobile ad-hoc wireless sensor networks. The main features and contributions of this paper are as follows. First, we use physical layer information of $R F$-energy harvesting when routes are selected in network layer. Second, we use energy aware entropy based mesh route selection strategy adaptively to efficiently support route stability. Third, we use a direction-guided line using location information to support energy saving for the establishment of routing routes in the given networks. The performance evaluation using OPNET shows that the proposed routing protocol can efficiently support PDR, average and minimum remaining energy of nodes, and the lifetime of routes and networks, respectively.
\end{abstract}

Keywords: mobile ad-hoc wireless sensor network, RF-energy harvesting, direction guided line, entropy, adaptive mesh route selection

\section{Introduction}

Sensor networks that can be established in tough environments such as mountains and seas that may not be easily accessible can transfer and give useful information to the administrators in monitoring centers. Recently, many researchers have been focusing on how to extend node lifetime and save energy consumption effectively. One research approach to solve those problems is to use RF-energy harvesting that uses RadioFrequency to charge the battery in the physical layer.

The authors in [1] study the impact of relay selection scheme in RF energy harvesting environment during data transmission. It was found that the increased energy harvesting time in data transmission decreases the probability of successful data transmission. Therefore, the proportion of energy harvesting in data transmission is an important fact. The authors in [2-3], on the other hand, try to optimize energy allocation and managements in RF energy harvesting. Meanwhile, the authors in [4-6] design and evaluate energy harvesting circuit, and present optimization. We confirm the need for a variety of studies in related works [1-6] while the related works [1-6] focus on physical layer.

On the other hand, the authors in [7] study a routing protocol in network layer using energy information. Their energy-harvesting model uses a solar panel instead of RFenergy harvesting. This research shows a comparison of increased lifetime to DD protocol [8] that does not consider energy to select route.

Cross layer is an escape from the independent layer of TCP/IP communication model while the cross-layer approach is used actively to improve performance of communication systems recently. The authors in [9] combine application layer, data-link layer, and 
physical layer together to efficiently improve the performance of multimedia transmission. Our previous work [10], on the other hand, proposes a routing protocol that takes energy harvesting and efficiency into consideration.

The main features and contributions of the proposed routing protocol, EHER, can be summarized as follows.

- Cross-layer concept: In the proposed routing protocol EHER, a cross-layer concept with both the physical layer and network layer to establish routing routes is used. The EHER utilizes RF-energy harvesting information generated by an RFenergy harvest model in the physical layer when routes are selected in the network layer. It means that it is possible to harvest and charge the signal power for transmission.

- $\quad$ Entropy based adaptive mesh route selection: In EHER, mesh routing routes are adaptively selected based on entropy concepts using information from RF-energy harvesting to efficiently support route stability with increased packet delivery rate (PDR). It means that the number of routes between two nodes can be changed adaptively according to the condition of route stability. For example, we can select multiple mesh routes between two nodes if there are bad routes with low route stability, while only one route can be selected if the stability of all of routes is very high between two routes.

- Direction guided line using location information: In EHER, a strategy of direction guided line between source node and destination node using location information is used to efficiently support energy saving, control over head, and finally packet delivery ratio (PDR).

The rest of the paper is arranged as follows. Section 2 presents the proposed routing protocol, EHER, with basic concepts and architecture, and algorithm of EHER. A performance evaluation of the proposed EHER is described in Section 3. Finally, the conclusions are given in Section 4.

\section{Proposed Routing Protocol: EHER}

\subsection{Basic Concepts and Architecture}

Figure 1 describes the basic concepts and architecture of the proposed routing protocol, EHER. In the given networks, mobile ad-hoc wireless sensor networks, $S$ is a source node that works as sink node called by gateway that is connected to the Internet and gathers information from sensor nodes in the networks. D is a destination node. As we can see in Figure 1, the proposed EHER consists of the following concepts and architectures such as a cross-layer approach to the use information from RF-energy harvesting, entropy based adaptive mesh route selection to efficiently support route stability with increased packet delivery rate (PDR), direction guided line using location information to efficiently support energy saving, control over head, and finally packet delivery ratio (PDR), respectively. 


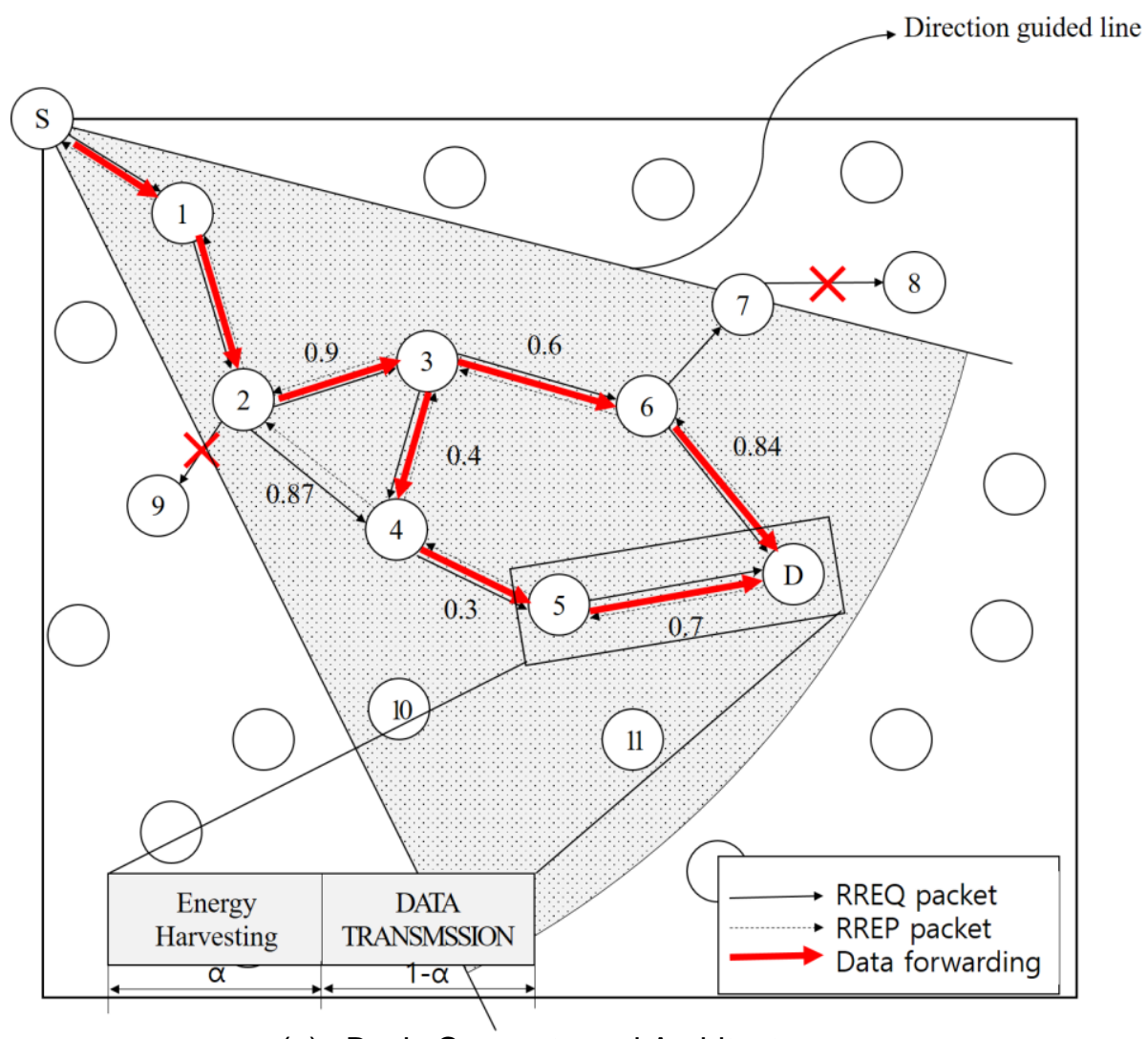

(a) Basic Concepts and Architecture

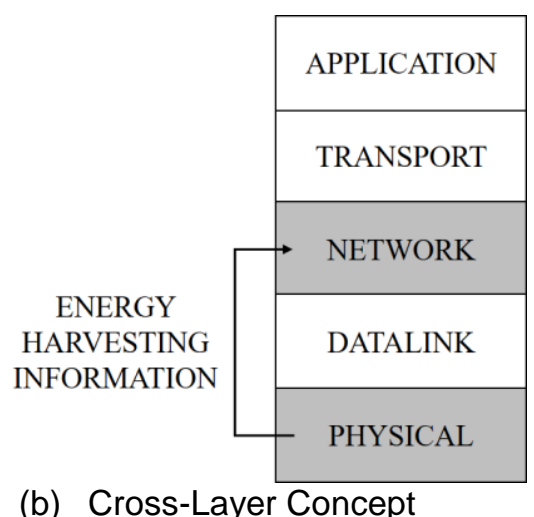

Figure 1. Basic Concepts and Architecture of Proposed EHER

\subsection{The Algorithm of Proposed Routing Protocol, EHER}

Figure 2 presents the operations of the proposed EHER algorithm. The operations of the EHER algorithm consists of the following: 


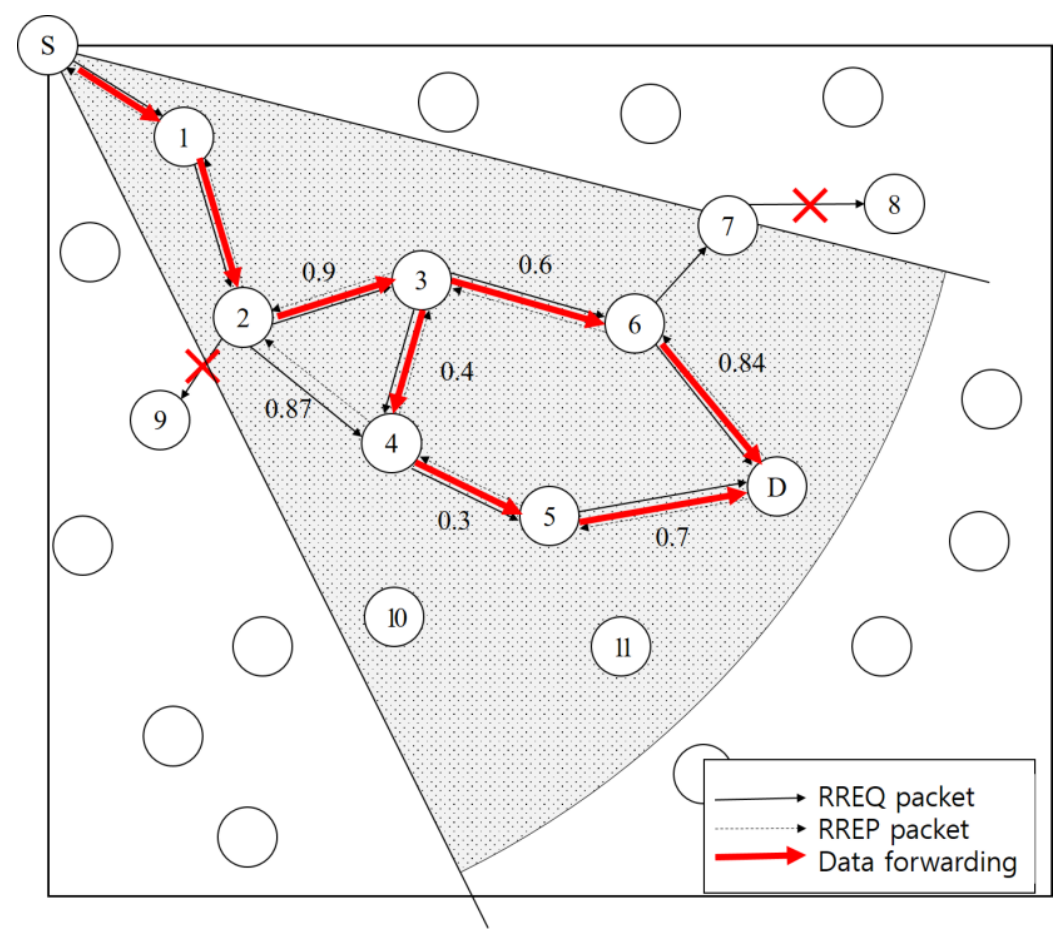

Figure 2. Operation of EHER Algorithm

Step 1: When an event is happening, a source node $S$ (sink) broadcasts Request packet (RREQ packet) to find a destination. The RREQ packet includes some information such as source ID, destination ID, previous node ID, current node ID, previos node energy information, hop-count, and direction guided line information to destination, current node energy information. Where node energy information means the remaining energy of node according to the RF-energy harvesting per unit session time. Node energy information can be updated and given by using Eq. (1) according to the amount of comsumed energy and RF-energy harvesting by node.

$$
e_{m}(t)=e_{m}(t, t+\Delta t)
$$

Where $e_{m}$ a function of updated energy at a node is, $t$ is time and $\Delta t$ is unit time interval. RF-energy harvesting uses RF signal to charge energy and transmit data. More specifically, transmission between source and destination is carried out in each fixed block time with two phases. In the first phase of $\alpha$, all nodes harvest energy from other nodes. In the second phase of $(1-\alpha)$, the node broadcasts its signal. The role of direction-guided line is to control the region range for route construction. In this paper, the direction guided line is fixed with angle $\theta=\pi / 3$.

Step 2: When a node receives the RREQ packet, the node checks and confirms whether the node is a destination node and within the direction guided line or not. If the recevied node is not a destination node and within the direction guided line, the node updates Request routing table (REQ_Table) and broadcasts RREQ packet to neighbor nodes. These operations are repeated until a destination node receives the RREQ packet. If a received node is not a destination node and out of the region range of the direction guided line, the node doesn't broadcast the RREQ anymore.

Step 3: When a destination node receives an RREQ packet, the destination node updates the REQ_Table by using the information in the RREQ packet. The destination node generates Reply packet (RREP packet), which consists of source ID, destination ID, previous ID, current node ID, previous node energy information, current node energy 
information, hop-count. The destination node selects allowed candidate mesh routes to previous nodes, then forwards RREP packet to the assigned previous nodes to source node via selected candidate mesh routes.

Step 4: When a node receives an RREP packet, the node calculates the route stability between the node (current node) and a destination node based on the entropy using the remaining energy information of each node as follows:

Calculates the probability of relative change of energy for neighborhood nodes at the current node $m$ on the selected candidate route by using Eq. (2)

$$
P_{m}(t)=\frac{e_{m}(t)}{\sum_{i \in F_{m}} e_{i}(t)}
$$

Where $F_{m}$ is a set of neighborhood nodes. Therefore, denominator means the summation of remaining node energy of neighborhood nodes.

We use Eq. (2) to calculate route stability between a current node and a destination. Route stability means the strength of route. Therefore, a much higher value of route stability means that this route can survive in much longer time in the energy viewpoints. Using Eq. (2), the entropy at the current node $\mathrm{m}$ is given by Eq. (3)

$$
E_{m}(t)=\frac{P_{m}(t) \log \left(P_{m}(t)\right)}{\mathrm{C}_{F_{m}}}
$$

Where $E_{m}(t)$ is entropy using energy information at a node $\mathrm{m}$ for the neighbor nodes on the selected candidate route. $\mathrm{C}_{F_{m}}$ is the number of neighbor nodes on the selected candidate route. $E_{m}(t)$ has the normalized value as $0 \leq E_{m}(t) \leq 1$.

The route stability between a current node and a destination node is obtained as Eq. (4) if there are $\mathrm{N}$ number of hop counts between a current node and a destination node.

$$
\text { Route_Stability }=\prod_{m=1}^{N} E_{m}(t)
$$

Route stability is strength of route. The high route stability means result of Eq. (3) is constant with the results in the route.

$>$ The current node updates Reply routing table (REP_table) by using the route stability information for all the selected candidate routes. In this case, the current node can adaptively select the number of mesh routes (ex: mesh routes or tree route) between the current node and a previous node to the destination node, according to the value of route stability.

$>$ These operations at the current node are repeated for all the selected candidate routes.

Step 5: When a source node (sink node) receives the RREP packet, first the source node repeats the operations in step 4 . Then, the source node starts to forward the data packet to the next node via selected routes (mesh routes or tree route) according to the route stability in the routing table (REP_table) to the destination node

\section{Performance Evaluation}

In this section, the performance of our proposed routing protocol, EHER, is evaluated by using OPNET. Table 1 describes simulation environments. 
Table 1. Simulation Environments

\begin{tabular}{|c|c|}
\hline Parameter & Descriptions \\
\hline Network Size & $1,000 \mathrm{~m} \times 1,000 \mathrm{~m}$ \\
\hline Radio Range & $250 \mathrm{~m}$ \\
\hline Number of Sink & 1 \\
\hline Number of Mobile Node & 50 \\
\hline \multirow{2}{*}{ Random Waypoint Mobility } & $0 \mathrm{~km} / \mathrm{h} \sim 60 \mathrm{~km} / \mathrm{h}$ \\
\cline { 2 - 2 } & Direction: $0 \sim 2 \pi$ \\
\hline Mobility Pause Time & $0 \sim 10 \mathrm{sec}$ \\
\hline Capacity of node battery & $45 \mathrm{~W}$ \\
\hline Transmitter Cost & $1.2 \mathrm{~W}$ \\
\hline Charge Rate & $0 \sim 1.5 \mathrm{~W}$ \\
\hline
\end{tabular}

The network size is $1 \mathrm{~km} \times 1 \mathrm{~km}$ and consists of 50 mobile nodes, and one sink in the mobile ad-hoc wireless sensor networks. The transmit range of each node is 250 $\mathrm{m}$ and the transmission direction of each node is $0 \sim 2 \pi$. We use the angle of direction guided line with a pre-specified region range value $\pi / 3$. We assume that all nodes already know their location information using GPS information. All nodes can move dynamically with random mobility. Random mobility means that node speed is changed according to uniform random distribution from 0 to maximum speed $60 \mathrm{~km} / \mathrm{h}$. Mobility pause time means the waiting time of a node to the next movement. Mobility pause time also is changed according to uniform random distribution from 0 to 10 seconds. Capacity of node battery is $45 \mathrm{~W}$ and one transmission consumes $1.2 \mathrm{~W}$ signal power for transmitter and receiver, respectively. Volume of RF energy harvesting is from 0 to $1.5 \mathrm{~W}$. This value is related to the node distance from transmitter to receiver.

Figure 3 shows a comparison of the packet delivery ratio (PDR) of proposed EHER with ADOV-EH [10] and AODV. PDR means how many packets can be transmitted from a source node to a destination node successfully.

\section{Packet Delivery Ratio}
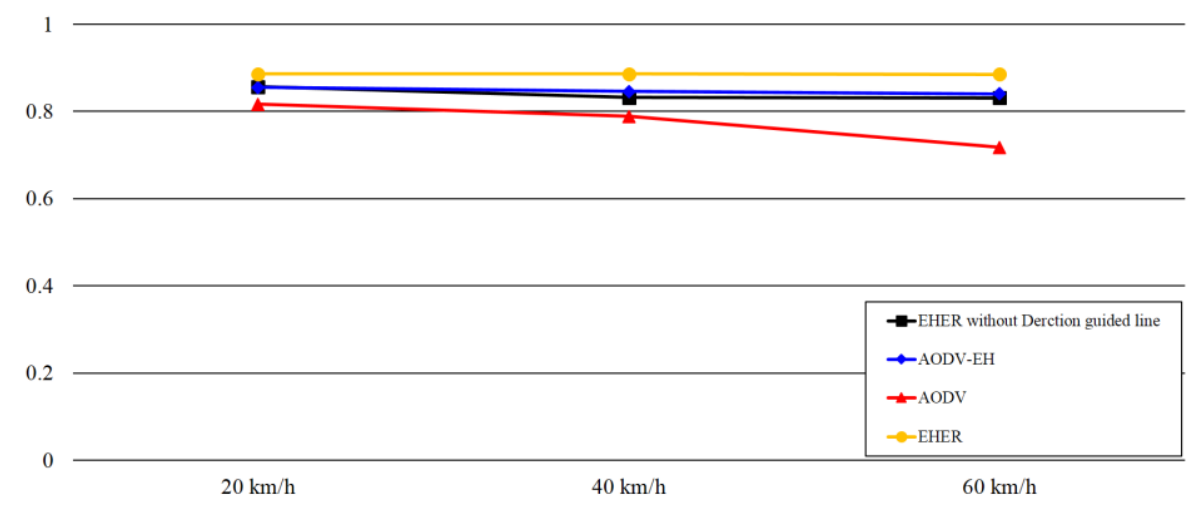

Figure 3. PDR as a Function of Node Speed 
As we can see in Figure 3, EHER can support PDR efficiently. The reason is that EHER can efficiently support the stability of a route by using RF-energy harvesting aware entropy-based concepts to establish adaptive mesh routes. Also, since EHER utilizes direction-guided line using location information, the number of control overhead and consumed energy for route construction can be reduced.

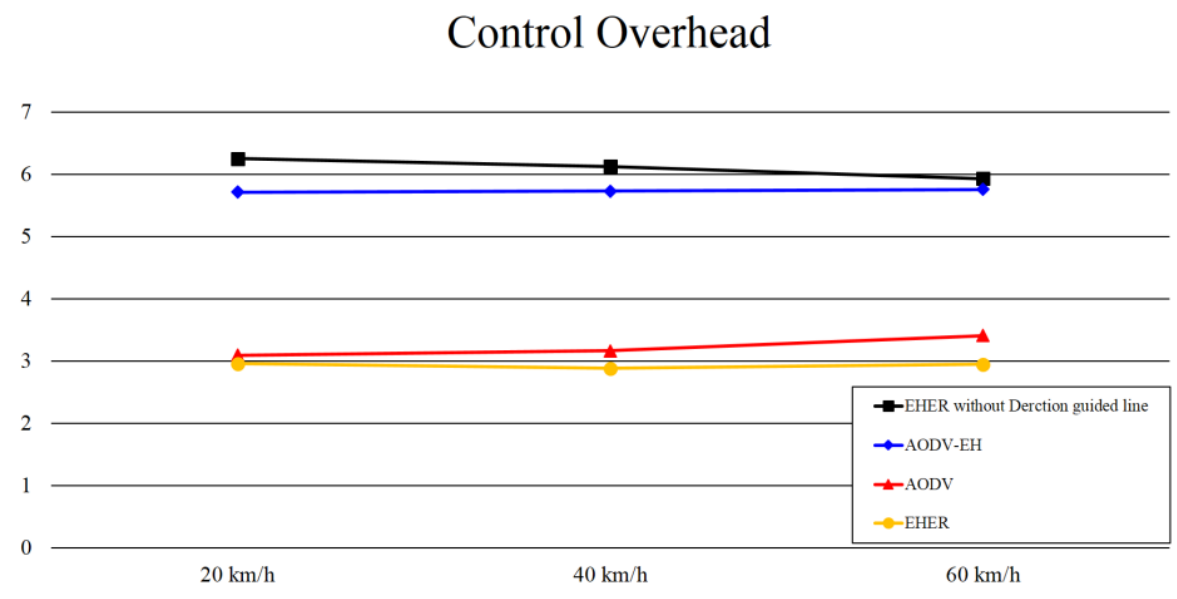

Figure 4. Control Overhead as a Function of Node Speed

Figure 4 shows a comparison of the control overhead of the proposed protocol, EHER, with AODV-EH and AODV. The control overhead means the number of control signal packets (RREQ, RREP) required per session setup for each node. As we can see in Figure 4, EHER can efficiently reduce the control overhead to establish routing routes. The reason is that a direction-guided line using EHER can reduce a large number of control signal packets to establish routing route. However, as we can see in Figure 4, if we do not use direction-guided line, the control overhead is increased because mesh routes require a lot of control signal packets to establish routing routes.

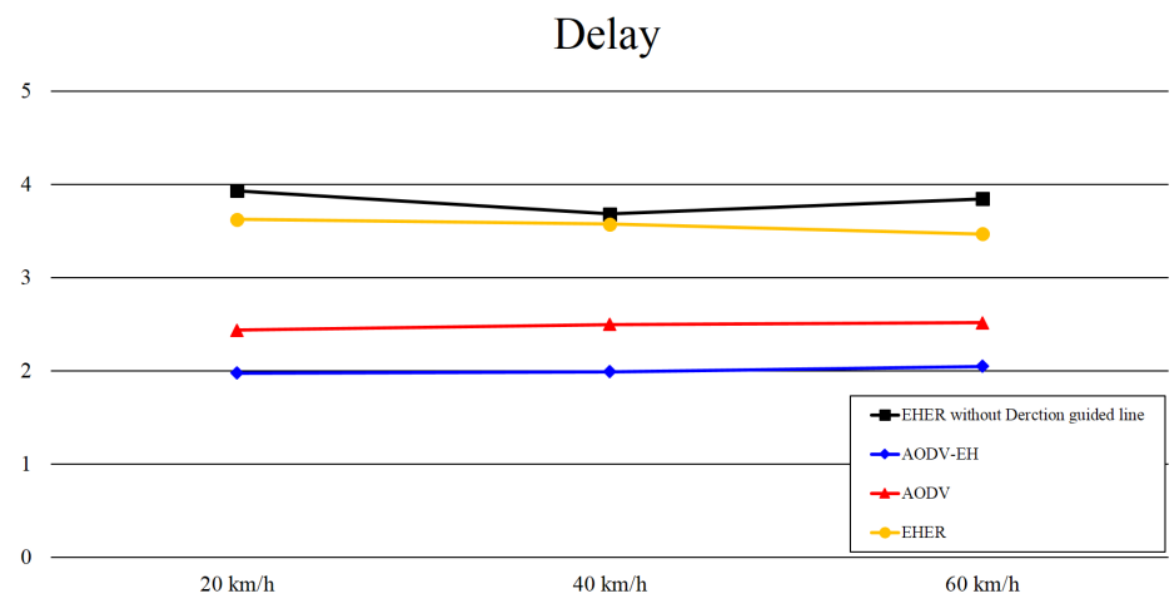

Figure 5. Delay as a Function Node Speed

Figure 5 shows a comparison of delay of the proposed EHER with AODV-EH and AODV. Delay is the consumed time for construction of routing routes session setup. As we can see in Figure 5, EHER has a bit of high delay. The reason is that EHER 
uses adaptive mesh routing routes, which require much time compared to the tree route for session setup for the construction of routing route.

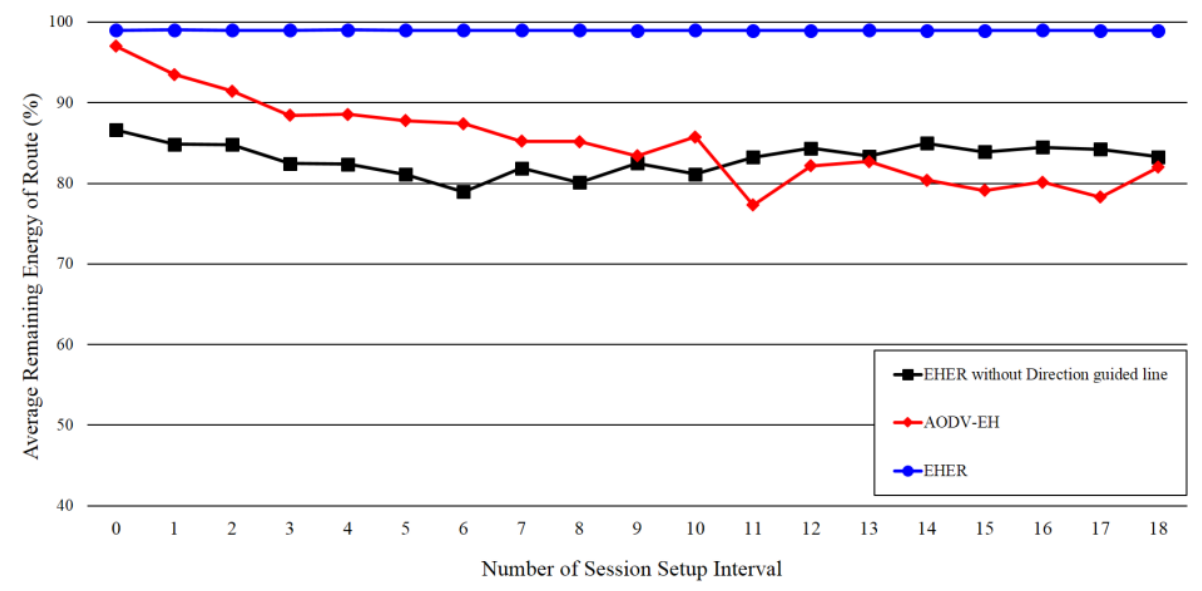

Figure 6. Average Remaining Energy of Node in the Selected Route

Figure 6 shows a comparison of the average remaining energy of a node in the selected route. As we can see in Figure 6, EHER can efficiently support average remaining energy of nodes in a selected route. The reason is that EHER can reduce the consumption of energy for construction of routing routes by using directionguided line and save energy by using RF-energy harvesting. As we can see in Figure 6, the average energy of EHER keeps around 97\%.

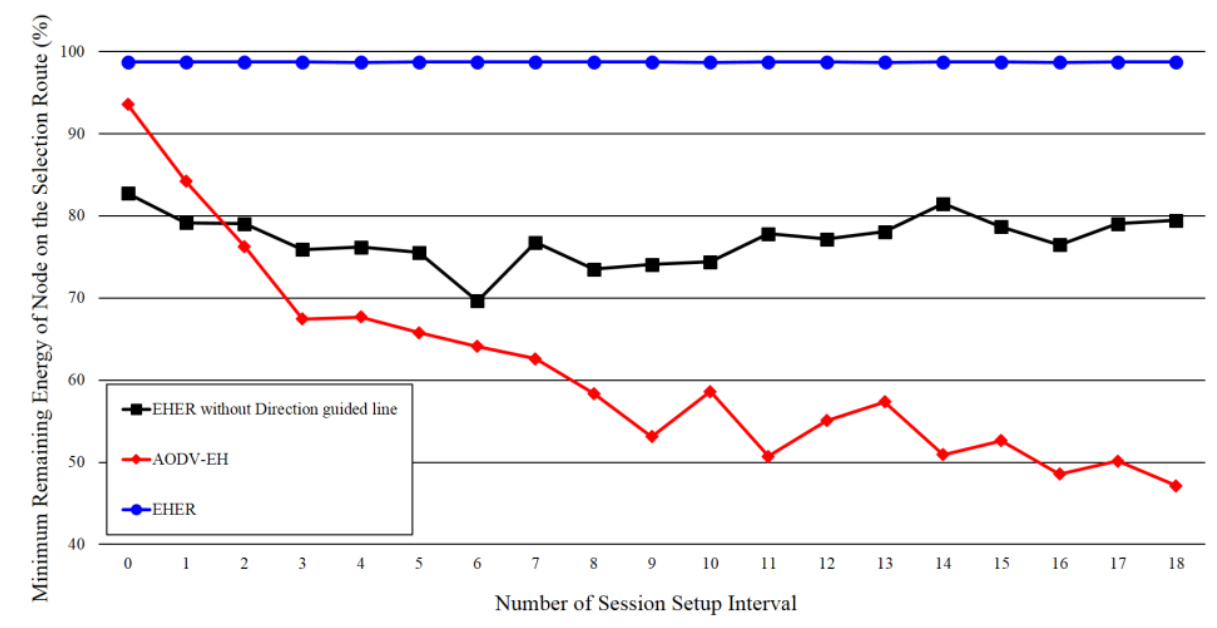

Figure 7. Minimum Remaining Energy of Node on the Selected Route

Figure 7 shows a comparison of the minimum remaining energy of nodes on the selected route. As we can see in Figure 7, EHER can efficiently support the minimum remaining energy of nodes on the selected route. The reason is that EHER can reduce the consumption of energy for construction of routing routes by using direction-guided line and save energy by using RF-energy harvesting. As we can see in Figure 7, EHER can keep a constant value of minimum remaining energy of node on the selected route to around $97 \%$. 


\section{Conclusion}

In this paper, we proposed an energy harvesting aware entropy based routing protocol, called EHER. The main features of the EHER were to use the following concepts such as cross-layer concept, entropy based adaptive mesh route selection, and direction guided line using location information to increase transmission efficiency. RF-energy harvesting was performed in the physical layer while the information of RF-energy harvesting was used for route construction in the network layer with cross-layer concepts. To select stable adaptive mesh routes quantitatively. An entropy concept using energy information was applied for selecting stable adaptive mesh routes quantitatively. Additionally, a strategy using location information called direction-guided line was introduced to efficiently support energy saving and control overhead. The performance evaluation showed that the proposed EHER could effectively support transmission efficiency with high PDR, low control overhead, low delay, and high remaining energy of node. In our next studies, we shall focus on how to support adaptive direction guided line according to route stability analytically.

\section{Acknowledgments}

This research was supported by the MSIP (Ministry of Science, ICT and Future Planning), Korea, under the ICT/SW Creative Research program (IITP-2015R2212150026) super-vised by the IITP(Institute for Information \& communication Technology

\section{References}

[1] N. T. Do, V. N. Q. Bao and B. An, "A relay selection protocol for wireless energy harvesting relay networks", Proceedings of 2015 International Conference on Advanced Technologies for communications (ATC), Ho Chi Minh City, Vietnam, (2015).

[2] C. K. Ho and R. Zhang, "Optimal energy allocation for wireless communications powered by energy harvesters", Proceedings of 2010 IEEE International Symposium on Information Theory, Austin, TX, (2010).

[3] V. Sharma, U. Mukherji, V. Joseph and S. Gupta, "Optimal energy management policies for energy harvesting sensor nodes", Journal of IEEE Transactions on Wireless Communications, vol. 9, no. 4, (2010), pp. 1326-1336.

[4] H. Jabbar, Y. S. Song and T. Jeong, "RF energy harvesting system and circuits for charging of mobile devices", Journal of IEEE Transactions on Consumer Electronics, vol. 56, no. 1, (2010), pp. 247-253.

[5] X. Cao, W. J. Chiang, Y. C. King and Y. K. Lee, "Electromagnetic Energy Harvesting Circuit with Feedforward and Feedback DC- DC PWM Boost Converter for Vibration Power Generator System", Journal of IEEE Transactions on Power Electronics, vol. 22, no. 2, (2007), pp. 679-685.

[6] P. Nintanavongsa, U. Muncuk, D. R. Lewis and K. R. Chowdhury, "Design Optimization and Implementation for RF Energy Harvesting Circuits", Journal of IEEE Emerging and Selected Topics in Circuit and Systems, vol. 2, no. 1, (2012), pp. 24-33.

[7] M. K. Jakobsen, J. Madsen and M. R. Hansen, "DEHAR: A distributed energy harvesting aware routing algorithm for ad-hoc multi-hop wireless sensor networks", Proceedings of 2010 IEEE International Symposium a World of Wireless Mobile and Multimedia Networks(WoWMoM), Montreal, QC, Canada (2010), pp. 1-9.

[8] C. Intanagonwiwat, R. Govindan, D. Estrin, J. Heidemann and F. Silva, "Directed Diffusion for Wireless Sensor Networking," Journal of IEEE/ACM Transactions on Networking, vol. 11, no. 1, (2003), pp 2-16.

[9] M. V. D. Schaar and N. S. Shankar, "Cross-layer Wireless Multimedia Transmission: Challenges, Principles, and New Paradigms", Journal of IEEE Wireless Communications, vol. 12, no. 4, (2005), pp 50-58.

[10] K. Shim and B. An, "An Energy Harvesting Aware Routing Protocol in Mobile ad-hoc Wireless Sensor Network", Proceedings of International Conference on Green and Human Information Technology (ICGHIT), Clark, Angeles City, Philippines, (2016). 


\section{Authors}
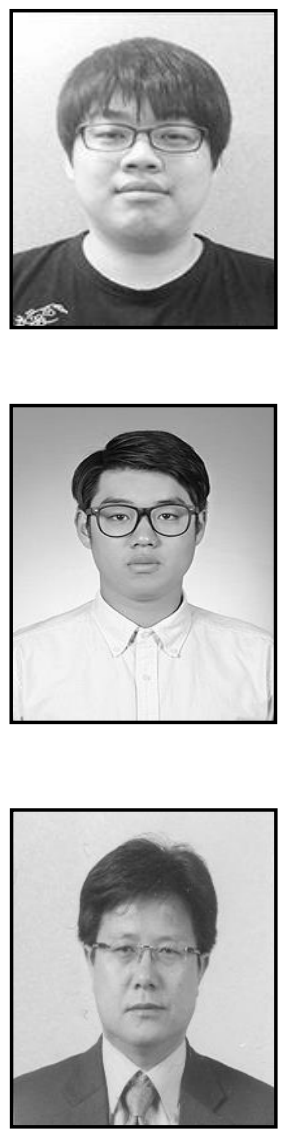

Kyuhyun Shim, he was born in Seoul, Republic of Korea. He received his B.S. in Computer and Information Communications Engineering from Hongik University in 2015. Since 2015, he has been working as aN M.S student in Information System Graduate School of Smart City Science Management, Hongik University, Republic of Korea. His major research interests are ad-hoc networks, sensor networks, wireless communications, and energy harvesting.

Kyusung Shim, he was born in Seoul, Republic of Korea. He received his B.S. in Computer and Information Communications Engineering from Hongik University in 2012. Since 2015, he has been working as an M.S student in Information System Graduate School of Smart City Science Management, Hongik University, Republic of Korea. His major research interests are wireless communications, cognitive radio and physical layer security.

Beongku An, he received his M.S. degree in Electrical Engineering from New York University (Polytechnic), NY, USA, in 1996 and Ph.D. degree from New Jersey Institute of Technology (NJIT), NJ, USA, in 2002, respectively. He received his B.S. degree in Electronic Engineering from Kyungpook National University, Korea, in 1988. From 2003, he joined the Faculty of the Department of Computer and Information Communication Engineering, Hongik University in Korea, where he is currently a professor. From 1990 to 1994, he was a senior researcher in RIST, Pohang, Korea. He was a lecturer and RA in NJIT from 1997 to 2002. In 2012, he worked as a president in IEIE Computer Society in Korea, and from 2011 to 2016 as a general chair in International Conference, ICGHIT, respectively. His current research interests include wireless networks and wireless communications such as ad-hoc networks, sensor networks, wireless internet, ubiquitous networks, and cellular networks, cognitive radio networks, mobile clouding, IoT, visible light communications (VLC). Professor An was listed in Marquis "Who's Who in Science and Engineering" and "Marquis Who in the World", respectively. 